\title{
Conceptos fundamentales en la formación de especialistas de la comunicación organizacional en algunas universidades mexicanas
}

Conceitos fundamentais na formação profissional de especialistas em comunicação organizacional em algumas universidades do México

Fundamental concepts in the professional training of organizational communication specialists in some universities in Mexico

\section{Eva María Pérez Castrejón}

Doctora en Comunicación Aplicada por la Universidad Anáhuac México.

Profesora de la Universidad Popular Autónoma del Estado de Puebla (UPAEP).

E-mail: evamaria.perez@upaep.mx

\section{Cristina Barroso Camiade}

- Doctora en Comunicación Aplicada por la Universidad Anáhuac México.

- $\quad$ Profesora de la Universidad Anáhuac México.

- E-mail: cristina.barrosoca@anahuac.mx 


\section{Resumen}

Como las tendencias de la comunicación organizacional van evolucionando y se van ajustando a las demandas de la globalización, se producen impactos naturalmente en la enseñanza de la disciplina y las organizaciones públicas y privadas. Este artículo pretende definir los conceptos fundamentales en la formación de especialistas de la comunicación organizacional utilizando metodología cualitativa mediante entrevistas a profundidad a los líderes de programas académicos y a empleadores para conocer las demandas del mercado y cómo las universidades las atienden.

\section{PALABRAS CLAVE: COMUNICACIÓN ORGANIZACIONAL•ESPECIALISTA DE COMUNICACIÓN ORGANIZACIONAL•ORGANIZACIÓN•EMPLEADORES.}

\section{Resumo}

Dia após dia, as tendências da comunicação organizacional evoluem e se ajustam às demandas da globalização, impactando, naturalmente, o ensino da disciplina e as organizações públicas e privadas. Portanto, este artigo tem como objetivo definir os conceitos fundamentais na formação profissional de especialistas em comunicação organizacional por meio de uma metodologia qualitativa e de entrevistas em profundidade com líderes de programas acadêmicos e empregadores, a fim de entender as demandas do mercado e a forma como as universidades as atendem.

PALAVRAS-CHAVE: COMUNICAÇÃOO ORGANIZACIONAL•ESPECIALISTA EM COMUNICAÇÃOO ORGANIZACIONAL•ORGANIZAÇÃO • EMPREGADORES.

\section{Abstract}

Day after day the trends of organizational communication are evolving and adjusting to the demands of globalization, naturally impacting the teaching of the discipline, and public and private organizations. Therefore, this article aims to define the fundamental concepts in the professional training of organizational communication specialists using a qualitative methodology by applying in-depth interviews to leaders of academic programs and employers to learn about the market demands and the way universities meet them. 


\section{INTRODUCCIÓN}

L a comunicación organizacional ha trascendido con una perspectiva nacional e internacional que no solo se queda en las aulas, sino que se traslada a organizaciones micro, pequeñas, medianas y grandes. Su alcance abarca tanto la enseñanza de la disciplina como su práctica tratando de atender a las demandas sociales, por lo que los especialistas se han dado a la tarea de responder por diferentes diagnósticos con acciones prácticas, análisis de casos, visitas a organizaciones y medios de comunicación que van desde los tradicionales hasta las redes sociales. Las respuestas de los expertos han permitido que la comunicación organizacional se fortalezca y esté a la vanguardia del desarrollo de esta era.

Los especialistas en comunicación organizacional proporcionan sus puntos de vista acerca de la disciplina: de cómo poner en práctica una mejor enseñanza y cómo llevar a la praxis en las organizaciones la comunicación organizacional. Estos actores son quienes experimentan en la organización, diseñan e implementan acciones o planes estratégicos los cuales impactan en la motivación, productividad e imagen de quienes integran las organizaciones. Es fundamental combinar la teoría y la práctica para poder explicar mejor las áreas de oportunidad de la organización, poder diseñar estrategias y piezas de comunicación acordes al sistema, saber interpretar las necesidades de las personas que están vinculadas a la organización de manera interna y externa, y, así, crear los mensajes con las mejores narrativas, por lo tanto, la comunicación organizacional llega a incidir en toda la organización y quienes la integran.

Ante esto, a lo largo de este texto se conocerá la opinión de los especialistas que lideran programas académicos, quienes mencionan los contenidos que en materia de comunicación organizacional consideran que deben ser incluidos en los temarios académicos para después ponerlos en práctica; también se conocerá la opinión de los empleadores de organizaciones públicas o privadas, quienes comparten los aspectos que toman en cuenta para poder contratar a egresados especialistas de la línea en comunicación organizacional, además se expondrán cuáles son los conceptos fundamentales en la formación profesional de los especialistas de la comunicación organizacional en México. Las variables que se estudian son las siguientes: Filosofía organizacional, cultura, vivencia cultural, comuniwcación estratégica, efectividad, técnicas, eficiencia, jerarquía, dirección, liderazgo, ética, ciudadanía, responsabilidad social organizacional y mejora social.

A partir de experiencias de los líderes de programas académicos y los empleadores, quienes narraron de manera descriptiva sus puntos de vista y opiniones de las variables antes mencionadas, se compartieron los conocimientos no solo teóricos, sino también prácticos, mostraron una realidad muy heterogénea desde las diferentes perspectivas, además se rescataron las ideas y conceptos más relevantes de los hechos que se describen. Dichos especialistas contribuyen a transmitir la importancia de la comunicación organizacional para el cometido, impulso y proyección de cualquier tipo de organización.

La comunicación organizacional posibilita desarrollar competencias comunicativas intencionadas y sistemáticas que dan apertura a responder a las diferentes áreas de oportunidad de comunicación en las organizaciones; esto es posible si está respaldado por bases teóricas que contribuyen a interpretar, en la práctica, los hechos de manera más efectiva y poder proponer herramientas que aporten a la consecución de los objetivos y a la solución de las áreas de oportunidad. Hay conceptos fundamentales en la formación de los especialistas de la comunicación organizacional en México para saber responder a las organizaciones públicas y privadas el qué, quién, cómo, cuándo, dónde y por qué implementar piezas de comunicación formales e informales.

\section{REFERENCIAL TEÓRICO}

La comunicación organizacional tiene sus orígenes en la década de 1920 gracias al desarrollo de la teoría de la organización. En este mismo periodo, a partir de los estudios realizados por los fundadores de la teoría de la comunicación Lasswell, 
Lazarsfeld, Hovland y Lewin se empieza a hablar de la existencia de "una comunicación interpersonal donde se puede identificar a una audiencia activa, organizada y segmentada que se encuentra comprometida con los valores, las creencias y las costumbres de los diferentes grupos a los que pertenece" (Alonso; Becerra, 2011, p.1); esto permite que se geste el concepto de comunicación intergrupal como antecedente de la comunicación organizacional.

Durante las últimas décadas del siglo XXI, el desarrollo de propuestas a nivel internacional dentro del campo de la comunicación organizacional ha favorecido el surgimiento de numerosos enfoques que han permitido el planteamiento de construcciones teóricas que sirven para explicar y comprender de una mejor manera este fenómeno. En México el estudio de la comunicación organizacional se ha enfocado en la diversidad de organizaciones que van desde la gran empresa, las pequeñas y medianas empresas, las organizaciones privadas y gubernamentales hasta las instituciones de educación superior. Lo anterior refleja que el estudio de los fenómenos organizacionales no siempre ha sido abordado desde una misma perspectiva, sino que ha permitido el desarrollo de la innovación organizacional, que es un tema altamente recurrido en el ámbito académico.

La comunicación organizacional en México debe tener en cuenta la disposición de un sistema de gestión del conocimiento, que sea puesto en práctica con la intención de que los miembros puedan crear, transferir e integrar conocimientos al servicio común en que se asegure el diálogo abierto y argumentado entre los miembros y partes. Esto requiere de un lenguaje común que permita un espacio de encuentro transparente, abierto y comprometido a la hora de ejercer sus funciones en la evolución de la organización como parte de un todo (Alonso; Becerra, 2011).

De acuerdo a Collado (1994), la comunicación organizacional ayuda a "desarrollar e implementar estrategias de comunicación que respalden a la organización en el logro de sus objetivos, ofreciendo recursos efectivos de coordinación, apoyando los procesos de cambio y reforzando la integración personal" (p.65). Para este autor, la comunicación organizacional es una herramienta que le permite a la organización alcanzar sus objetivos mediante el diseño de estrategias que se encuentran alineadas con el propósito que la organización ha definido para sí misma.

Para Abraham Nosnik (2013), la comunicación en las organizaciones es un concepto más profundo y debe estudiarse como una teoría sistémica de comunicación al interior de las organizaciones, además explica que debe de ser abierta, evolutiva, flexible, multidireccional e instrumentada. Por otro lado, Corella, Toledo y Moreno (2011) mencionan que la comunicación organizacional debe ser vista como una comunicación integral que permita la producción, procesamiento e intercambio de información, que internamente y desde un sistema económico, político, cultural o social se dé a la tarea de hacer fluir y acelerar mensajes e interacciones entre todos sus grupos de interés, a partir de tres puntos: público interno y externo; comunicación corporativa y relaciones públicas; y la comunicación mercadológica.

Hasta ahora queda de manifiesto que este tipo de comunicación se encarga de los flujos de mensajes de la organización tanto con sus públicos internos como con los externos y que se encuentra conformada por una serie de estrategias que buscan mejorar esa circulación de información. De lo anterior hay que resaltar que una buena definición de los objetivos y el uso de tácticas adecuadas no van a garantizar su éxito, lo que debe existir es una identidad y una cohesión al interior de la organización que se vea reflejada en la imagen que se tiene de la empresa al exterior, para que entonces estos mensajes sean exitosos y la lleven a cumplir sus objetivos.

Lo anterior nos lleva a repensar la responsabilidad que tienen las instituciones de educación superior en la preparación de los futuros profesionales de la comunicación organizacional para que sean capaces de ayudar a la organización en la consecución de sus objetivos. En otras palabras, es necesario que estos expertos cuenten con el perfil requerido por los empleadores y las organizaciones, cualquiera que sea la naturaleza de estas. Esto evidencia el hecho de que la comunicación organizacional está compuesta por recursos material y humano, es decir, conjunta un perfil profesional con el desarrollo del individuo dentro de la misma organización, con la intención de provocar mejores ambientes organizacionales. 


\section{METODOLOGÍA}

En este apartado se presenta el diseño metodológico que se utilizó para saber cuáles son los conceptos fundamentales en la formación del profesional de la comunicación organizacional en México, desde la perspectiva de los líderes de los programas académicos y de los empleadores. Fueron seleccionados estos dos grupos, ya que los primeros son los responsables de diseñar los contenidos de las asignaturas que les permitirán a los alumnos desarrollar las habilidades necesarias para desenvolverse como especialistas de la comunicación organizacional. Pero también era importante tomar en cuenta la voz de los empleadores, porque conocen las necesidades no solo de la empresa, sino del mercado en términos de comunicación organizacional. Consideramos que ambos puntos de vista son necesarios e indispensables para contar con profesionales altamente capacitados dentro del mercado laboral.

Las preguntas que se plantean dependen de tres factores: la longitud de la entrevista, la naturaleza de las preguntas y la naturaleza de la investigación. Las entrevistas fueron audiograbadas y se hizo notas de las mismas para conocer la opinión de empleadores y de los líderes de programas académicos de la Universidad Popular Autónoma del de Puebla (UPAEP) y de la Universidad Autónoma del Estado de Hidalgo (UAEH), siendo la primera privada y la segunda pública, en cuanto a qué conocimientos deben tener los egresados especialistas de la línea en comunicación organizacional. En las entrevistas que se realizaron, se siguió una guía de tópicos que establece el orden de los cuestionamientos realizados durante los encuentros.

Esta investigación se realiza con empleadores y líderes de los programas académicos de las Licenciaturas en Comunicación 0 Ciencias de la Comunicación de la UPAEP y la UAEH. Las universidades antes mencionadas fueron seleccionadas debido a que los planes educativos contemplan la formación de especialistas en este campo.

Previo a la entrevista se dialogaron los temas que serían tratados (filosofía organizacional, cultura organizacional, comunicación estratégica, técnicas, jerarquía, ética y responsabilidad social), con el fin de controlar los tiempos y posibles dispersiones por parte del entrevistado. Las estructuras de las preguntas se elaboraron a partir de los objetivos que se buscaban alcanzar con la investigación. Se partió de una introducción en que el entrevistador expresó el propósito de la entrevista, su estructura y qué obtiene. Se hizo del conocimiento de los entrevistados que la información obtenida se analizaría con atención y cuidado, atendiendo en todo momento la confidencialidad de los datos. Se realizaron seis entrevistas en total, dos a líderes de programas académicos y cuatro a empleadores de estos estas licenciaturas.

A continuación, se presentaron las principales aportaciones de los líderes de programas académicos.

\section{Percepción de los líderes de programas académicos}

Se debe mencionar que, para llegar a estos resultados, se utilizó un análisis textual; y en esta investigación los entrevistados serán citados con las siglas: entrevistado uno EPELPAK 1 (UPAEP) y entrevistado dos EPELPAE 2 (UAEH).

Para EPELPAK 1, la filosofía organizacional se encuentra ubicada dentro de la cultura organizacional, se aborda a partir de la creación de significados compartidos, permitiendo identificar los recursos simbólicos que dan valor a lo que es cultura organizacional. La cultura organizacional es un concepto que aporta a la organización, que se crea, se mantiene y se puede evaluar a partir del grado de identidad que los colaboradores tienen hacia la organización. Tal concepto se traduce en misión, visión, valores, ideología, símbolos y creencias, como valores que son fundamentales, que dan valor agregado a la organización, por lo que es fundamental que los educandos lo trabajen por medio de los proyectos de intervención. 
En cuanto a la comunicación estratégica, EPELPAK 1 mencionó que es un conocimiento fundamental en el educando, ya que supone la formación técnica y le da la posibilidad de conocer los objetivos y metas de las organizaciones y cómo coadyuvar a la realización de estos a través de la comunicación. Como parte de los conceptos fundamentales que deben poseer los educandos de la disciplina se encuentran: comunicación organizacional, comunicación estratégica, cultura organizacional, clima organizacional, competencias y habilidades, manejo de crisis, responsabilidad social organizacional y técnicas metodológicas. Tales conceptos favorecerán el aprendizaje y el desarrollo de herramientas y técnicas de trabajo.

En lo que respecta a las técnicas, el entrevistado uno refirió que son importantes las cualitativas y las cuantitativas, ya que contribuyen a la elaboración de los proyectos de intervención. Los proyectos necesitan de técnicas de capacitación, talleres, comunicación productiva, manejo de crisis, comunicación cara a cara, trabajo en equipo, dinámicas de grupo. Dichas técnicas no siempre se pueden abordar en el aula por cuestión de tiempo aun cuando son clave para la construcción y aplicación de planes estratégicos de comunicación.

De acuerdo con EPELPAK 1, otro tema importante que debe ser incluido en los programas académicos es el concepto de jerarquía en las organizaciones, ya que permite tener un panorama general de lo que es la estructura de una organización desde su representación gráfica, la generación de flujos de comunicación y el camino que siguen. El tema de estructura en las organizaciones también contribuye al diseño de los proyectos de intervención. La ética es un componente esencial que deben contener las guías de aprendizaje y del especialista de la comunicación organizacional. Dado que profesionalmente se enfrenta a diferentes tipos de conflictos o crisis, la ética es lo que distingue y le da valor al profesional de la comunicación.

No se puede hablar de ética sin responsabilidad social organizacional. Ambos conceptos que se dan a conocer en el aula son parte fundamental en la formación del profesional de la disciplina, en el acompañamiento del maestro con los alumnos, en la construcción de proyectos de intervención no solo para generar productividad, sino valor agregado a través del recurso humano. Finalmente, EPELPAK 1 mencionó que todo especialista de la comunicación organizacional debe conocer conceptos, como clima organizacional, cultura organizacional, competencias comunicativas, manejo y prevención de crisis, diseño, ejecución y evaluación de proyectos de comunicación organizacional, responsabilidad social y comunicación estratégica.

A continuación se presentan las principales contribuciones del EPELPAE 2. Para él, los contenidos como filosofía e ideología organizacional son importantes dentro de los contenidos de la licenciatura en comunicación, pero a este nivel es complicado incluirlos, ya que la comunicación organizacional va a un enfoque más pragmático, así se está descuidando la construcción teórica y se trata de centrarse más en el estudio de la comunicación como una ciencia. Abraham Nosnik en los años 1990 comenzó a hablar de la comunicación organizacional como una presencia constante en las organizaciones, donde se puede estudiar a la filosofía organizacional como una cuestión pragmática. A diferencia de Rafael Ávila que menciona que este concepto más que práctico es saber integrar a la gente a una organización. Esta reflexión permite argumentar que a partir de sexto semestre es cuando un especialista en comunicación organizacional debe conocer conceptos como filosofía organizacional e ideología.

EPELPAE 2 indicó que los contenidos de aprendizaje deben abordarse por categorías, estructuras y análisis. Esto permite saber cómo está funcionando la organización, quiénes son sus integrantes y cómo se relaciona a los miembros, además los educandos necesitan conocer cómo elaborar modelos de intervención para saber cómo resolver problemas. Conceptos como misión, visión, valores, ideología, símbolos y creencias permiten al estudiante comprender la esencia del concepto de cultura organizacional. Este concepto al ser tan profundo requiere un conocimiento teórico antes de llevarlo a la práctica y actuar por sentido común. De acuerdo al entrevistado dos, por acciones de ese tipo se llegan a confundir conceptos; por ejemplo, ya que la comunicación estratégica es un concepto fundamental en la formación de especialistas en comunicación organizacional, se debe integrarla a conocimientos como cultura, estrategia y valores.

Por otro lado, EPELPAE 2 mencionó que este tipo de comunicación contribuye a la solución de problemas, aunque no de manera inmediata lleva un proceso que se apoya de estrategias que permiten detectar, planear y ejecutar el plan de acción. Por lo tanto, 
ANO 18 • NÚMERO 37 • SETEMBRO / DEZEMBRO $2021 \cdot$ ORGANICOM

CONCEPTOS FUNDAMENTALES EN LA FORMACIÓN DE ESPECIALISTAS DE LA COMUNICACIÓN ORGANIZACIONAL EN ALGUNAS UNIVERSIDADES MEXICANAS

una asignatura referente a estrategias debe estar durante todo el programa, y el especialista debe saber sobre temas de jerarquía y dirección en la organización, ya que se trabaja con una estructura organizacional que tiene diferentes roles de mando y toma de decisiones. Además, apuntó que la concepción de liderazgo ayudará a que los estudiantes puedan detectar conceptos como clima organizacional y cultura organizacional. La ética en el especialista de la comunicación es uno de los problemas con los que más se enfrenta en las universidades, ya que se cree que este concepto se adopta en casa, y no siempre es verdad, por lo que es necesario que este tema se incluya en la guía de estudios, actualmente se aborda como ética y comunicación. Es necesario ser ético, honesto y tener valores en cada actividad que se aplique en la organización.

A continuación se presentan los resultados de las entrevistas que se realizaron a los empleadores de los programas académicos de la UPAEP y de la UAEH.

\section{Percepción de los empleadores de los programas académicos}

Para fines de esta investigación los entrevistados serán citados con las siglas: EPEEUI1, EPEEUA 2, EPEEHCM 1 y EPEEHMM 2.

El entrevistado EPEEUI1 mencionó lo esencial que es para un egresado de la disciplina en comunicación organizacional el conocer temas de filosofía en las organizaciones y de cultura organizacional como elementos estratégicos de toda organización. Puntualizó que un especialista en comunicación organizacional contribuye de manera estratégica a las organizaciones, generalmente quien viene realizando esta tarea son mercadólogos aun cuando no tienen la capacidad para redactar mensajes claros y establecer objetivos concretos que puedan ser comprendidos por cualquier nivel jerárquico.

De igual forma apuntó que el egresado en la disciplina aplica técnicas de capacitación, talleres, comunicación productiva, manejo de crisis, relaciones cara a cara, trabajo en equipo, dinámicas de grupo y tiene la capacidad de analizar cualquier tipo de mensaje. También es un líder que guía a los empleados e impulsa los cambios en las organizaciones que dependen de otras jerarquías. El especialista sabe persuadir para poder llegar con sus mensajes a todos los niveles. Finalmente, EPEEUI1 indicó que la ética es un componente que no puede perder de vista el especialista de la comunicación organizacional, ya que tiene contacto permanente con personas y él es ejemplo de lealtad con la empresa; esta junto con la responsabilidad social impacta en la mejora y crecimiento de quienes integran a la organización.

De acuerdo al reclutador EPEEUA 2, un egresado interesado en realizar ejercicios profesionales en comunicación organizacional necesita conocer lo que es la filosofía en las organizaciones, ya que va acorde a lo que necesita comunicar en la organización y para el establecimiento de objetivos. Con lo que respecta a la cultura organizacional, el rol del egresado es estratégico, pues las organizaciones tienen hoy en día una función multinacional, no se pueden establecer objetivos si no se conoce cuál es la esencia de la organización que está reflejada a través de su cultura.

Finalmente, EPEEUA 2 indicó que quien práctica la comunicación organizacional da el rumbo, se convierte en el pilar de la organización y busca el bienestar de un grupo; mantiene los valores internos y externos que facilitan los flujos de comunicación. El egresado es quien identifica las problemáticas, las grandes oportunidades de los equipos de trabajo, favorece el flujo de comunicación e idea procesos o herramientas para mejorar los ambientes laborales; ejerce liderazgo en el momento de interactuar en presentación de proyectos, de manera personal y en el momento de establecer y fijar objetivos. La ética siempre debe estar presente en las personas y para poder impulsar estrategias de responsabilidad social organizacional.

Por otro lado, los entrevistados de la UAEH refirieron lo siguiente: EPEEHCM 1 señaló que la filosofía organizacional es la pieza de la que depende el funcionamiento de una organización, es parte de su identidad, la guía de valores y lenguajes que se comparten e 
induce el compromiso y la competitividad en la labor de los colaboradores. Indicó, además, que cuando el egresado aplica estrategias de cultura organizacional, contribuye a una función estratégica, ya que esto permite que se cumplan normas y reglas, eficacia en los proyectos, asimismo involucra valores como la honestidad y trabajo en equipo con la intención de favorecer la interacción entre los miembros de una organización.

Señaló también que el especialista en este campo aporta herramientas de comunicación estratégica, que permiten alcanzar los objetivos de la organización; aplica técnicas de tipo interpersonal, es decir, cara a cara, y trabajo en equipo que permiten la retroalimentación, es un "aprender-aprender" en que se mejoran las habilidades, los conocimientos y las conductas. El liderazgo del egresado de la disciplina lo refleja en relaciones personales y el trabajo en equipo, sin embargo, de manera contraria y no bien comprendido el liderazgo se ve reflejado cuando existe la lucha de poderes. El líder de una organización debe influir en las demás personas, inspirar, motivar y dirigir.

Para terminar, EPEEHCM 1 explicó que la ética y la Responsabilidad social organizacional son temas de impacto organizacional, pues estos conceptos requieren la participación de cada miembro de la organización y permiten que se adquieran compromisos y una responsabilidad compartida.

Por otra parte, EPEEHMM 2 mencionó que el concepto de filosofía en las organizaciones es necesario para el egresado no solo en nivel básico, sino a mayor profundidad. En lo que respecta al concepto de cultura organizacional en una sociedad como la mexicana, es poco aplicable y usable. Cuando el especialista de la comunicación organizacional realiza acciones de comunicación estratégica, está pensando en el exterior, en el cómo vender, vincular y promover a la empresa, ya que a nivel interno en la organización es poco valorado, pues los directores lo consideran como un gasto innecesario.

Por otro lado, puntualizó que el problema actual del comunicólogo es que ingresa a los trabajos como camarógrafos profesionales y no como profesionales de la comunicación que saben de manejo de crisis o de mejoramiento de la productividad. En las organizaciones, los empleados desconocen sus beneficios y obligaciones, hay bajos rendimientos de productividad y altos niveles de rotación de personal, por lo que es importante que el egresado aplique estrategias de comunicación interna que puedan arreglar estos problemas.

Por último, mencionó que la ética es un componente esencial en todo lo que se hace, la sociedad necesita urgentemente un replanteamiento de la ética y los valores que posibilite cambios positivos en un futuro. La responsabilidad social organizacional no es funcional si el director de la organización no lo hace vivo como una filosofía emprendedora.

En el siguiente apartado se desarrollarán los resultados que se obtuvieron de las aportaciones de las seis entrevistas que se realizaron.

\section{RESULTADOS}

En este apartado se presenta el análisis interpretativo por variable de las seis entrevistas que se llevaron a cabo en esta investigación, y los resultados se muestran agrupando las respuestas de los líderes académicos con las de los empleadores para plantear conclusiones más claras y pertinentes.

\section{Filosofía organizacional}

Los informantes señalan que la filosofía organizacional se encuentra ubicada en la cultura organizacional, es la creación de significados compartidos, se enfoca al entorno real y profesional y está centrada en los recursos humanos. El docente no puede 
enseñar valores organizacionales, sino que enseña filosofía de la organización. La filosofía organizacional es un concepto que va más allá de la praxis, ya que favorece una estructura intelectual y racional; por lo que se aprende más en el trabajo diario que en los libros, pues es un elemento estratégico de toda organización. Se necesita comunicar en la organización para el establecimiento de objetivos. El concepto de filosofía organizacional no debe abordarse en un nivel básico, sino a mayor profundidad y más que un enfoque pragmático se debe abordar desde la construcción teórica.

\section{Cultura organizacional}

Los entrevistados citan que la cultura organizacional se crea, se mantiene y se evalúa, se traduce en misión, visión, valores, ideología, símbolos y creencias, lo que permite al estudiante comprender la esencia de este concepto. Es necesario conocer este término primero en la teoría antes de llevarlo a la práctica. La cultura organizacional comprende a la organización en su sentido abstracto, el saber qué es y qué proyectar de manera interna o externa. Este concepto permite a los educandos tener un conocimiento más diverso y una visión más estratégica de las organizaciones, una función multinacional y establecer objetivos. Sin embargo, es poco aplicable y usable en México.

\section{Comunicación estratégica}

Los informantes refieren que la comunicación estratégica es un conocimiento fundamental en el educando, supone la formación técnica y da la posibilidad de conocer los objetivos y metas de las organizaciones. Como asignatura debe ser transversal en las diferentes materias, ya que permite saber cómo se gestiona e implementa, identifica, previene y soluciona una crisis organizacional. Este término integra conocimientos como cultura, estrategia y valores, induce a la creación de mensajes claros y establece objetivos concretos; es pensar en el exterior, en el cómo vender, vincular y promover a la empresa.

\section{Técnicas}

En voz de los informantes las técnicas en la disciplina de la comunicación organizacional deben ser cualitativas y cuantitativas, se pueden reflejar en acciones específicas como talleres, comunicación productiva, manejo de crisis, comunicación cara a cara, trabajo en equipo, dinámicas de grupo, como parte de la construcción y aplicación de planes estratégicos de comunicación. Las técnicas son un concepto teórico y práctico que tienen que ver con la comunicación productiva ya que permiten arreglar problemas organizacionales.

\section{Jerarquía}

Sobre esta variable los entrevistados mencionan que permite tener un panorama general de lo que es la estructura de una organización, siendo necesaria para la toma las decisiones. El liderazgo es una técnica que se utiliza en el trabajo, hay tipos de líderes y el especialista puede contribuir a crearlos dentro de la organización para después generar estrategias de comunicación. Conocer el concepto de jerarquía permite reafirmar en los educandos la necesidad de aprender a tomar decisiones de carácter ejecutivo; también les da la posibilidad detectar conceptos como el clima organizacional y cultura organizacional.

Los educandos necesitan aprender a desarrollar su rol de líder y la importancia de la ética en esta práctica. Sumado a lo anterior el liderazgo es importante en el egresado para sus actividades de producir, organizar y persuadir para poder llegar a todos los niveles. Se ve reflejada la acción del líder en el momento de presentar proyectos, en interacciones personales y en el momento de establecer y fijar objetivos. Muchas veces los especialistas de la comunicación organizacional no se sienten líderes cuando es una herramienta que impulsa los cambios en las empresas. 


\section{Ética}

Los informantes hablan de la ética como un concepto que no se puede desprender de la práctica profesional, es ejemplo de lealtad con la empresa, parte esencial de las personas e impulsa a la implementación de estrategias de responsabilidad social organizacional. Al ser un componente esencial en todo lo que hace el ser humano, posibilita cambios positivos en un futuro sustentados en valores. Además, constituye en un problema con el que más se enfrenta en las universidades, por lo que es necesario que este tema se incluya en las guías de estudios como elemento que impulse valores y honestidad. Hoy en día desafortunadamente la ética no es un componente que caracterice a los egresados.

\section{Responsabilidad social organizacional}

La responsabilidad social organizacional de acuerdo a los entrevistados no puede existir sin la ética, ya que es un ejercicio de toda organización que contribuye a mantener un impacto positivo o negativo. Este concepto se apoya en leyes que rigen las relaciones con el cliente y los asuntos ambientales, permite cambiar al mundo y a la empresa, ya que impacta en la mejora y el crecimiento de quienes integran a la organización. Desafortunadamente falta desarrollar conocimientos y llevarlos a la práctica en relación con la persona y con el medio ambiente. No es funcional si el director de la organización no lo hace vivo como una filosofía emprendedora.

A continuación se presentan los cuadros 1, 2 y 3 que hacen un resumen del análisis de cada una de la variables que fueron utilizadas para desarrollar las entrevistas tanto con empleadores como con líderes de programas académicos.

Cuadro 1: Análisis, empleadores UPAEP

\begin{tabular}{|c|c|}
\hline Filosofía organizacional & $\begin{array}{l}\text { Elementos estratégicos de toda organización } \\
\qquad \text { Identidad }\end{array}$ \\
\hline Cultura organizacional & $\begin{array}{c}\text { Función estratégica } \\
\text { La honestidad y trabajo en equipo }\end{array}$ \\
\hline Comunicación estratégica & $\begin{array}{l}\text { Objetivos } \\
\text { Vender, vincular y promover }\end{array}$ \\
\hline Técnicas & $\begin{array}{l}\text { Capacitación, talleres, comunicación productiva, manejo de crisis, } \\
\text { relaciones cara a cara, trabajo en equipo, dinámicas de grupo }\end{array}$ \\
\hline Jerarquía & $\begin{array}{l}\text { Impulsa los cambios } \\
\text { Influir en las personas, inspirar, motivar y dirigir }\end{array}$ \\
\hline Ética & $\begin{array}{c}\text { No puede perder de vista } \\
\text { Compromisos y responsabilidad compartida } \\
\text { Cambios positivos }\end{array}$ \\
\hline Responsabilidad social organizacional & $\begin{array}{l}\text { Crecimiento de quienes integran } \\
\text { Compromisos y responsabilidad compartida }\end{array}$ \\
\hline
\end{tabular}

Fuente: Elaborado por las autoras. 
Cuadro 2: Análisis, empleadores UAEH

\begin{tabular}{|c|c|}
\hline Filosofía organizacional & $\begin{array}{c}\text { Identidad } \\
\text { Concepto profundo }\end{array}$ \\
\hline Cultura organizacional & $\begin{array}{c}\text { Función estratégica } \\
\text { Poco aplicable y usable }\end{array}$ \\
\hline Comunicación estratégica & Objetivos \\
\hline Técnicas & Comunicación interpersonal \\
\hline Jerarquía & Influir, inspirar, motivar y dirigir \\
\hline Ética & Compromisos y responsabilidad compartida \\
Cambios positivos
\end{tabular}

Fuente: Elaborado por las autoras.

Cuadro 3: Análisis interpretativo de empleadores de cada universidad

\begin{tabular}{|c|c|}
\hline \multirow{2}{*}{ Filosofía organizacional } & Elementos estratégicos de toda organización \\
Identidad \\
Concepto profundo \\
Aprendizaje acertado \\
No hay conocimiento
\end{tabular}




\section{CONSIDERACIONES FINALES}

A manera de conclusión se puede mencionar que es importante que los especialistas de la comunicación organizacional en México cuenten con determinados conceptos esenciales durante su formación profesional, lo que se ha reafirmado en una investigación cualitativa apoyada en entrevistas con líderes de programas académicos y empleadores.

Son necesarios e imprescindibles los conceptos de ética y responsabilidad social organizacional en el desarrollo profesional de los especialistas de la comunicación organizacional de México, además de conocimientos en filosofía organizacional, cultura organizacional, comunicación estratégica, técnicas y jerarquía. Por lo que es necesario crear una propuesta pedagógica en la que se establezca la importancia de estos contenidos teóricos que los especialistas de la disciplina desarrollen en la práctica. Es evidente que las universidades tanto públicas como privadas en el país tienen un interés por los procesos internos.

Igualmente se demuestra que la comunicación organizacional es una tarea integral e integradora para los especialistas quienes desarrollan competencias integrales entre la teoría y la práctica, lo que crea un reto porque suele ser complicado romper con paradigmas pedagógicos. Este concepto provoca que los actores que intervienen construyan una visión bien definida, que permee en las estructuras de las organizaciones y le den un valor agregado a las actividades que realizan.

Al mismo tiempo los individuos que integran las entidades académicas tienen el encargo de comprender que la comunicación organizacional en México vive una realidad compleja, por lo que hay una responsabilidad muy grande en la construcción y emisión de mensajes e información que se da en el aula. Estos tienen que ser el punto de partida para despertar el interés de los especialistas para hacer sus propias construcciones conceptuales, juicios y criterios que son puestos en práctica cuando interactúen en espacios de prácticas profesionales.

El manejar y tener muy claros determinados conceptos teóricos permite que el especialista de este campo contribuya con la toma de decisiones y sepa qué responder ante cualquier tipo de cuestionamiento. Esto sin perder de vista la ética como una forma de respeto a la dignidad humana y al bien común. Además, dado que el conocimiento de filosofía organizacional para ejecutar una acción es la base y la razón de ser de las organizaciones, es fundamental conocerlo en la teoría para llevarlo a la práctica, pues sin dicho concepto sería imposible alcanzar los objetivos organizacionales.

Además de los contenidos ya mencionados, es importante que la comunicación organizacional aporte a la formación del especialista de esta disciplina al visualizar que esta va más allá de la creación de piezas tangibles o intangibles de comunicación, ya que es un área de reflexión, que necesita propuestas con argumentos éticos que pongan de manifiesto que los valores son permanentes y se verán reflejados en cada una de las acciones que se realicen en la organización.

También se concluye que este campo ocupa un papel relevante para el estudiante de la disciplina, ya que para las organizaciones públicas y privadas de México se han convertido en una necesidad, de este modo la comunicación organizacional contribuye a la solución de conflictos o propone soluciones ante las áreas de oportunidad que se detecten. Esta disciplina necesita que se lleve a la práctica por un profesional que tenga prospectiva y que esté interesado en los cambios globales, en los avances tecnológicos y, sobre todo, en el recurso humano. El soporte de la comunicación organizacional radica en la estructura y la intención de los mensajes, necesita de la razón y la reflexión, con la intención de encausar esta práctica como un servicio a clientes internos y externos.

La disciplina estudiada de acuerdo a los entrevistados es una manera para alcanzar objetivos que están íntimamente relacionados con la filosofía organizacional, los cuales se hacen vivos a través de su cultura organizacional, por lo que 
el empleador busca especialistas que estén a la vanguardia de las nuevas tendencias de la disciplina y las nuevas tecnologías que permiten a la organización replantearse o reforzar la identidad como fuente de sentido, teniendo como referente principal la ética y la responsabilidad social organizacional. El empleador ve que el especialista de este campo es quien aporta un buen sistema de comunicación que proporciona la dirección de los clientes internos en apego al objetivo organizacional y afianza las relaciones de cooperación con la organización. Así la organización está obligada a estar permanentemente informada de lo que sucede dentro y fuera de ésta, ya que la información es el referente que contribuye a impulsar un mayor nivel de competitividad y desarrollo en cada una de las funciones, además de hacer que las organizaciones sean más productivas.

\section{REFERENCIAS}

ALONSO, Maria del Carmen T.; BECERRA, José Alberto. Surgimiento y desarrollo de la comunicación organizacional y su relación con otras ciencias. Revista Contribuciones a las Ciencias Sociales, Málaga, n.13, 2011.

COLLADO, Carlos Fernández. La comunicación en las organizaciones. Ciudad de México: Trillas, 1994.

CORELLA, Maria Antonieta Rebeil; TOLEDO, Jorge Alberto Hidalgo; MORENO, Mariana M. Gestión de la comunicación integrada en las organizaciones: competencias básicas para la formación del gestor. In: DUARTE, Gustavo Adolfo (coord.). Estudios de la comunicación: estrategias metodológicas y competencias profesionales en comunicación. Ciudad de México: Pearson Educación, 2011. p.51-82.

NOSNIK, Abraham. Teoría de la comunicación productiva: exploraciones más allá de la retroalimentación. Santa Fe: Homo Sapiens, 2013.

Artículo recibido el 24.06.2021 y aprobado el 27.09.2021. 